\title{
Widespread transduction of astrocytes and neurons in the mouse central nervous system after systemic delivery of a self- complementary AAV-PHP.B vector
}

\author{
Melvin Y. Rincon ${ }^{1,2} \cdot$ Filip de Vin ${ }^{1,2} \cdot$ Sandra I. Duqué ${ }^{1,2} \cdot$ Shelly Fripont ${ }^{1,2} \cdot$ Stephanie A. Castaldo $^{3,4}$. \\ Jessica Bouhuijzen-Wenger ${ }^{1,2} \cdot$ Matthew G. Holt ${ }^{1,2}$
}

Received: 28 June 2017 / Revised: 23 January 2018 / Accepted: 30 January 2018

(c) Macmillan Publishers Ltd., part of Springer Nature 2018

\begin{abstract}
Until recently, adeno-associated virus 9 (AAV9) was considered the AAV serotype most effective in crossing the blood-brain barrier (BBB) and transducing cells of the central nervous system (CNS), following systemic injection. However, a newly engineered capsid, AAV-PHP.B, is reported to cross the BBB at even higher efficiency. We investigated how much we could boost CNS transgene expression by using AAV-PHP.B carrying a self-complementary (sc) genome. To allow comparison, 6 weeks old C57BL/6 mice received intravenous injections of scAAV2/9-GFP or scAAV2/PHP.B-GFP at equivalent doses. Three weeks postinjection, transgene expression was assessed in brain and spinal cord. We consistently observed more widespread CNS transduction and higher levels of transgene expression when using the scAAV2/PHP.BGFP vector. In particular, we observed an unprecedented level of astrocyte transduction in the cortex, when using a ubiquitous CBA promoter. In comparison, neuronal transduction was much lower than previously reported. However, strong neuronal expression (including spinal motor neurons) was observed when the human synapsin promoter was used. These findings constitute the first reported use of an AAV-PHP.B capsid, encapsulating a scAAV genome, for gene transfer in adult mice. Our results underscore the potential of this AAV construct as a platform for safer and more efficacious gene therapy vectors for the CNS.
\end{abstract}

\section{Introduction}

Adeno-associated viruses (AAV) have proved to be one of the most promising viral vector systems for use in gene therapy, due to their excellent safety profile (non-replicative and non-pathogenic), ability to infect both dividing and

These authors contributed equally: Melvin Y. Rincon, Filip de Vin.

Electronic supplementary material The online version of this article (https://doi.org/10.1038/s41434-018-0005-z) contains supplementary material, which is available to authorized users.

Matthew G. Holt

Matthew.Holt@kuleuven.vib.be

1 VIB-KU Leuven Center for Brain \& Disease Research, Leuven, Belgium

2 KU Leuven, Department of Neuroscience, Leuven, Belgium

3 VIB-KU Leuven Center for Cancer Biology, Leuven, Belgium

4 KU Leuven, Department of Oncology, Leuven, Belgium non-dividing cells, and to provide long-term expression of any given transgene. AAV vectors have been especially useful for the treatment of neurological disorders in preclinical animal models-and this has led to numerous therapeutic trials in humans [1].

A major challenge when developing gene therapy for the central nervous system (CNS) is the route of delivery. Most neurological disorders need large areas of the brain to be targeted, if not the entire CNS. Therefore, direct intraparenchymal delivery, which has the advantage of requiring relatively low doses of vector, is not clinically appealing, as it is a highly invasive procedure, which usually leads to poor dispersion of the AAV vector and hence the transgene. Another alternative is to deliver AAV vector into the cerebrospinal fluid via intracerebroventricular or intrathecal (cisterna magna or lumbar puncture) injection. Various groups have shown the feasibility and therapeutic potential of this approach in rodent and large animal models [2]. However, this route of delivery is not risk free: the injection procedure requires highly trained personnel and can lead to infection and/or neurotoxicity [3]. 
From a clinical standpoint, systemic delivery remains ideal, as a single injection allows targeting of the entire CNS without complicated and invasive injection procedures - and their associated risks. A breakthrough came with the discovery that AAV vectors based on serotype 9 are able to cross the blood-brain barrier (BBB) and efficiently transduce CNS cells after systemic delivery [4, 5]. Importantly, the therapeutic benefit of this strategy was proven in various rodent models of neurological conditions [6-9]. However, several practical hurdles remain in relation to using systemic AAV delivery to treat CNS conditions. Principal amongst these are challenges in terms of vector production and safety, as high doses are generally required to achieve therapeutically relevant levels of transgene expression, following systemic administration. Thus, developing strategies that enhance overall levels of transgene expression in the CNS (or in specific CNS cell types) remains a priority. The development of self-complementary (sc) AAV-based vectors has proved effective in boosting transgene expression [10]. Various reports have shown that the use of a scAAV vector dramatically enhances expression of a given transgene (10 to 50-fold) in the CNS after systemic delivery $[4,11,12]$. Careful selection of promoters and regulatory elements can also boost expression in specific CNS cell types. The ubiquitously expressed CAG promoter (and its derivatives $\mathrm{CBA}$ and $\mathrm{CB}$ ), as well as the CMV promoter, are the most frequently used in both preclinical and clinical studies-as both promoter types give efficient transgene expression across all major CNS cell types [1]. However, the level of transgene expression varies considerably between cell types (both in terms of the number of cells expressing the transgene and the strength of expression), and this also depends on the specific regulatory elements used [13].

Off-target transduction represents another challenge when using a systemic delivery route. The use of CNSspecific promoters only partially addresses this issue [14]. Complementary approaches to reducing off-target transduction aim at enhancing vector tropism for the desired organ or cell type, while (simultaneously) reducing tropism for off-target systems. De novo synthesis of novel AAV capsids, or modification of existing capsids (through directed evolution [15], mutagenesis of specific amino acids in the capsid [16], or incorporation of specific targeting peptides [17]) have all proved to improve CNS transduction, while decreasing transduction in peripheral organs. In 2016, Deverman et al. [18] reported a significant advance in capsid design, when they published a selection method called Cre recombination-based AAV targeted evolution (CREATE) - which allows selection of AAV capsids that efficiently transduce defined Cre-expressing cell populations in vivo. They used CREATE to generate AAV capsids that efficiently transduce the adult mouse CNS after intravenous injection. One variant in particular, AAV-PHP. $\mathrm{B}$, was reported to transduce the majority of astrocytes and neurons across multiple CNS regions following systemic injection, leading to a 40-fold increase in transgene expression compared to AAV9, when both vectors used single-stranded expression cassettes. However, it is widely known that transgene expression using AAV9 is greatly enhanced by using a scAAV genome configuration (see above) [10]. Hence, in this study, we sought to further improve transduction levels in the CNS obtained with PHP. B. Using injections in adult mice, we show that expression levels, following systemic injection, are boosted when the PHP.B capsid envelops a self-complementary genome, and that these transduction levels exceed the current state of the art. As such, this vector configuration represents a substantial improvement over existing AAV-derived vectors for efficient and safe CNS use.

\section{Results and discussion}

To assess the effect of different genome configurations on the levels of CNS transduction obtained with the PHP.B capsid, we injected $1 \times 10^{11}$ vector genomes $(\mathrm{vg})$ of either ssAAV2/PHP.B-CBA-GFP or scAAV2/PHP.B-CBA-GFP in $\mathrm{C} 57 \mathrm{BL} / 6$ adult mice. As expected, based on knowledge of the AAV replication cycle [10], we observed higher levels of transgene expression in all brain regions evaluated with injection of scAAV2/PHP.B $(5516 \pm 587$ vs. $3442 \pm$ 420 a.u.; $\mathrm{p}=0.0074$ : Supplementary figure 1). Furthermore, our results revealed that the cell tropism was similar between the evaluated groups. The higher level of overall transgene expression obtained using the scAAV genome was largely the result of an increased number of astrocytes expressing GFP, relative to neurons (Supplementary figure 2). These results provide unambiguous confirmation that scAAV2/PHP.B gives superior CNS transduction following systemic injection.

\section{Systemic delivery of scAAV2/PHP.B-CBA-GFP achieved widespread transgene expression in the brain}

To obtain insight into the degree of improved BBB crossing and cell transduction possible with scAAV2/PHP.B-CBAGFP, we referenced it to the current gold standard, scAAV2/9-CBA-GFP (hereafter referred to as scAAV2/9), following intravenous delivery. Adult C57BL/6 mice were injected through the lateral tail vein at post-natal day 42 $\left(1 \times 10^{12} \mathrm{vg} /\right.$ mouse $)$. Transduction efficiency was evaluated in brain tissue using immunofluorescence analysis, 3 weeks postinjection. As previously reported, both viral vectors were able to cross the BBB and mediate GFP expression in 
various regions of the brain. However, GFP expression in mice injected with scAAV2/PHP.B was significantly increased compared to those that received scAAV2/9. Strong GFP expression was present in all major brain areas, including (but not limited to) cortex, striatum, hippocampus, brainstem and cerebellum (Fig. 1a, b). Quantitative analysis of GFP intensity in the different brain regions confirmed that scAAV2/PHP.B had higher transduction efficiency than scAAV2/9 in the cerebrum $(2105 \pm 161 \mathrm{vs}$. $1441 \pm 99$ a.u.; $p=0.0032)$, cerebellum $(2601 \pm 196$ vs. $1737 \pm 135$ a.u.; $p=0.0032)$ and brainstem $(3082 \pm 319$ vs. $2485 \pm 88$ a.u.; $p=0.0038$ ) (Fig. 1c).

We also investigated whether intracerebroventricular delivery of the scAAV2/PHP.B vector in adult mice could mediate widespread transgene expression, and whether the enhanced transduction efficiency observed with the PHP.B serotype (compared to scAAV9) was preserved using this route of delivery. Mice received a unilateral intracerebroventricular injection of scAAV2/9 or scAAV2/PHP.B $\left(4 \times 10^{9} \mathrm{vg} / \mathrm{mouse}\right)$, and GFP expression in the CNS was analyzed 3 weeks later (Supplementary figure 3 ). For both vectors, strong GFP signal was detected around the ventricle at the injection site and in the hippocampus. In particular, both the Cornus Ammonis sub region 3 (CA3) and CA1 of the hippocampus showed strong GFP expression. Lower levels of expression were observed throughout the entire CNS, with GFP-positive cells detected in cortex, thalamus, brainstem and cerebellum. However, no significant difference in transduction efficiency was observed between the two serotypes using this route of delivery (Supplementary figure 3). These results highlight that the enhanced transduction seen with the PHP.B capsid, following systemic delivery, most likely results from improved BBB crossing, rather than an increased ability to transduce cells per se.

\section{Systemic delivery of scAAV2/PHP.B carrying a CBA promoter mainly gave efficient transgene expression in astrocytes}

To identify the different cell types transduced after systemic delivery of scAAV2/PHP.B, we performed co-immunostaining using antibodies against GFP and specific CNS cell markers: $\mathrm{S} 100 \beta$ (astrocytes), Iba1 (microglia), CC-1 (oligodendrocytes), and NeuN (neurons) (Fig. 2). Most of the GFPexpressing cells in the cortex, brainstem and thalamus were astrocytes (Fig. 2a and data not shown), with fibrous astrocytes in the white matter and protoplasmic astrocytes in the gray matter being equally transduced. Manual counting of double-labeled cells (S100 $\beta$ and GFP) in the cortex showed that scAAV2/PHP.B significantly increased the number of astrocytes transduced in comparison to standard scAAV2/9 $(37.9 \pm 7.4 \%$ vs $12.8 \pm 3.1 \% ; p<0.001)$. The levels of astrocyte transduction observed with scAAV9 were similar to those reported previously $[11,19]$. However, neuronal transduction could only be observed in specific brain regions, such as the CA3 of the hippocampus and in the cerebellum (Fig. 2b). We did not observe microglia expressing GFP after delivery of either vector, in any of the evaluated regions. Hence, these data support the fact that scAAV2/PHP.B significantly enhances CNS transduction over and above the levels achievable with scAAV9 (when using equivalent promoters and transgene cargo).

We then evaluated transduction levels in the spinal cord (Fig. 3). Similar to the results we observed in the brain, scAAV2/PHP.B injection resulted in a higher level of GFP expression in the spinal cord, when compared to an equivalent dose of scAAV2/9. For both vectors, most of the GFP-expressing cells were astrocytes; only occasionally were motor neurons expressing the transgene observed, which is in direct contrast to previous reports $[14,18]$. Quantitative evaluation of GFP fluorescence confirmed a significant increase in fluorescence in the group injected with the PHP.B capsid $(2359 \pm 267$ vs $1639 \pm 113$ a.u.; $p=$ 0.001; Fig. 3c).

Taken together, these data show a pronounced bias towards efficient expression of the transgene in astrocytes, when scAAV2/PHP.B-CBA-GFP is administered by intravenous injection.

\section{Systemic delivery of scAAV2/PHP.B increases the number of transduced cells without modifying cell specificity}

We then performed a dose escalation comparison between the two vectors. Mice received a tail vein injection of $1 \times$ $10^{12} \mathrm{vg}$ (low dose) or $2.5 \times 10^{12} \mathrm{vg}$ (high dose) of AAV vector (scAAV2/PHB.B or scAAV2/9). GFP expression in CNS tissues was examined 3 weeks postinjection. Immunofluorescence analysis showed a dose-dependent increase in the level of cell transduction for both scAAV2/PHB.B and scAAV2/9, in all the CNS regions evaluated (Fig. 4a, b). As expected, quantitative analysis of fluorescence intensities showed increased GFP expression in the scAAV2/PHP.B group compared to scAAV2/9 (cerebrum $3731 \pm 420$ vs. $2571 \pm 141$ a.u.; $p=0.0001$ : spinal cord $4113 \pm 437$ vs. $2025 \pm 131$ a.u.; $p=0.0001$; Fig. 4 c). Of note, we did not see a change in the cell types targeted when using the highest vector dose. Similar to that observed with the low dose, the majority of transduced cells were astrocytes, with some GFP-positive neurons visible in hippocampus and cerebellum (not shown). Together with the results shown in Supplementary figure 2, these data strongly support the idea that the self-complementary genome configuration shows a strong cell type bias in transgene expression-with the increase in GFP fluorescence seen with dose escalation proportional to an increased number of 
Fig. 1 scAAV2/PHP.B vector gives better CNS transduction than scAAV2/9 when delivered systemically. C57BL/6 mice received a single tail vein injection of either scAAV2/9 or scAAV2/PHP.B vector, expressing the reporter gene GFP under control of the CBA promoter. The dose was $1 \times 10^{12}$ $v g$ in each case. GFP expression was analyzed 3 weeks postinjection. Representative coronal brain sections were immunostained for GFP.

Sections show various regions of (a) cerebrum and (b) cerebellum and brainstem. All brain regions from scAAV2/PHP.B injected mice showed stronger GFP expression than when scAAV2/ 9 was used. c Quantification of the relative fluorescence intensities (10 sections per mouse; 3 mice per group analyzed). $* * p<0.01$. a.u. arbitrary units. Scale bars: 1000 $\mu \mathrm{m}$
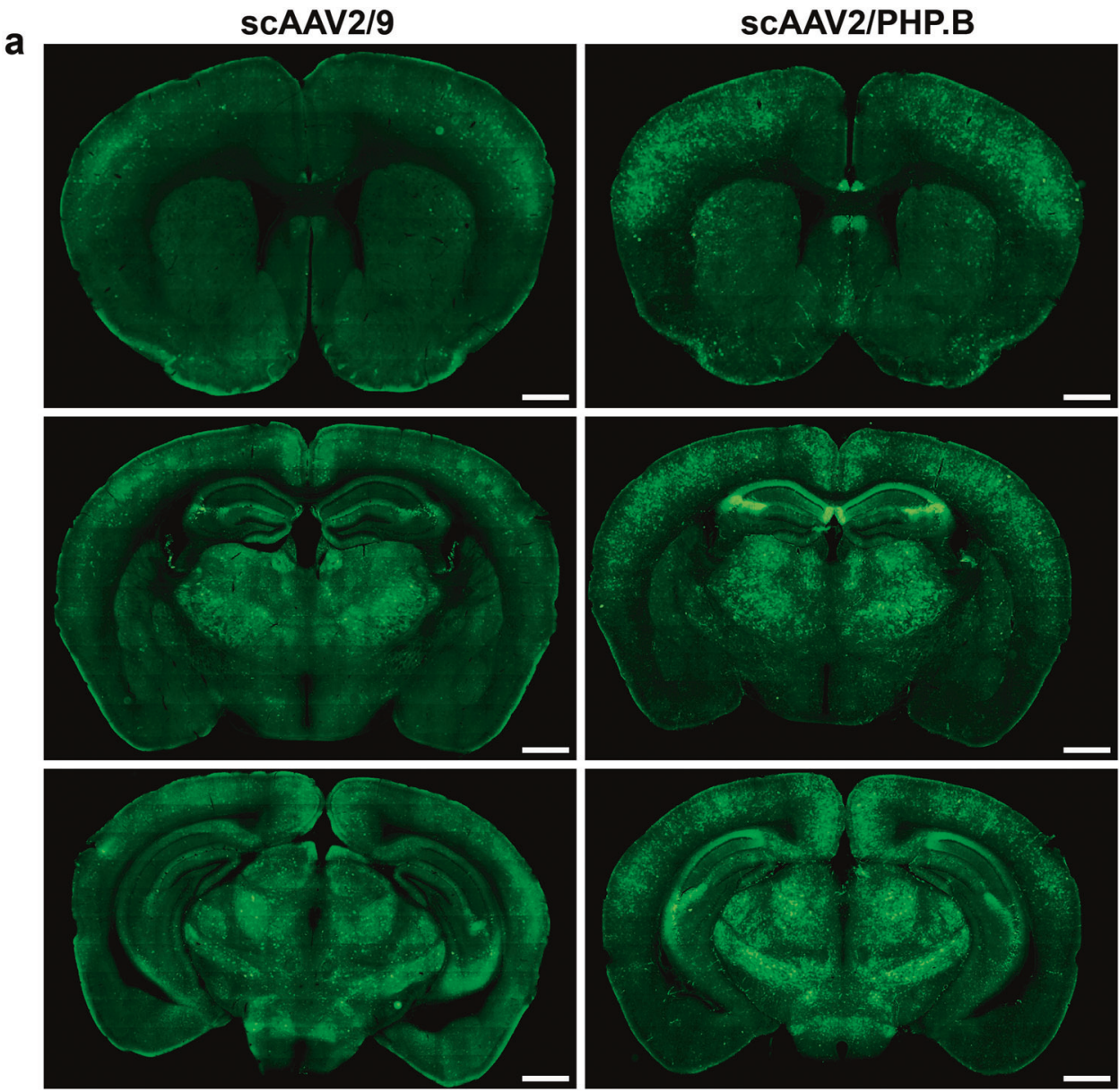

b
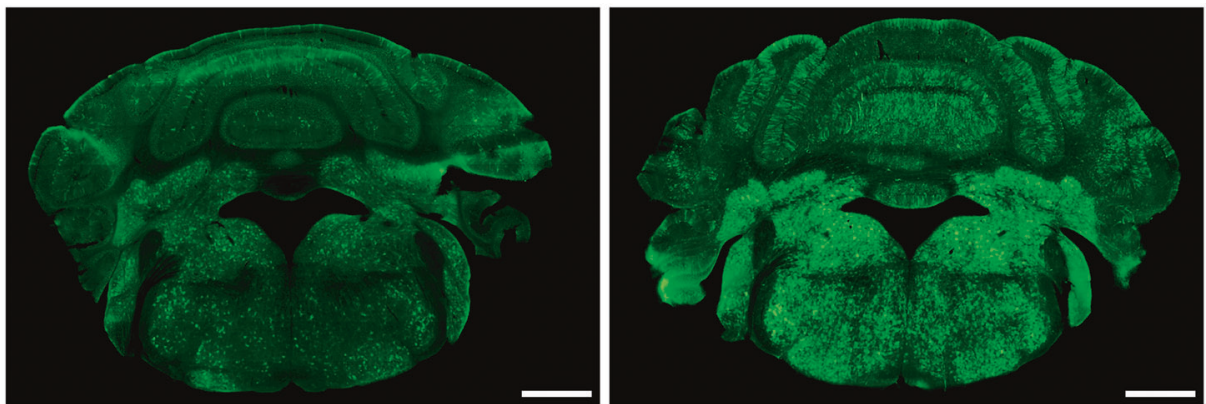

C
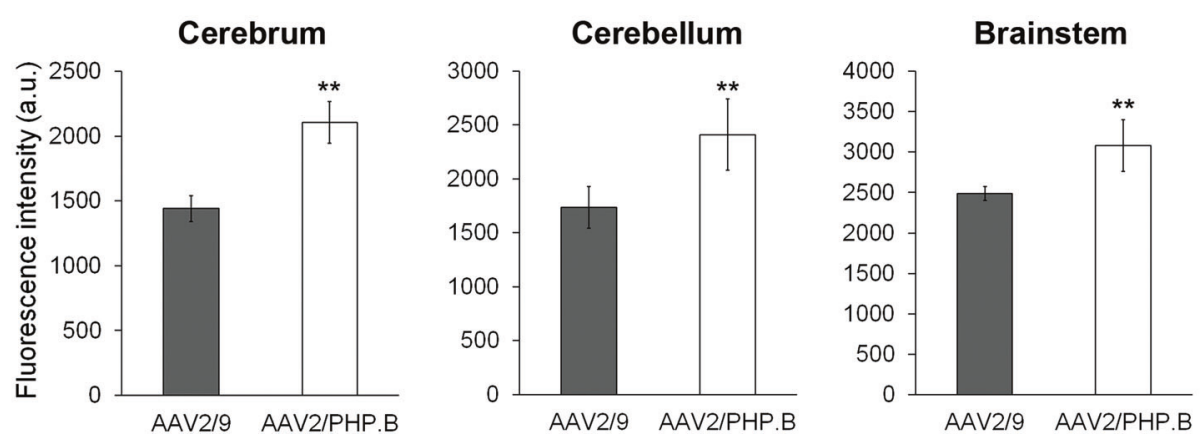
Fig. 2 scAAV2/PHP.B mostly transduces astrocytes in the brain following systemic delivery. Brain sections were immunostained for GFP (green) and cell markers specific for major CNS cell types (magenta). a In the cortex, most GFPexpressing cells were astrocytes (S100ß). A few GFP-expressing neurons (NeuN) were found. Oligodendrocytes (CC-1) were rarely transduced. We did not observe transduction of microglia, as judged by colocalization of GFP with Iba1. (Arrowheads indicate doublelabeled cells). b Representative images from the CA3 region of the hippocampus and the cerebellum, showing higher levels of neuronal GFP expression in these brain regions. Scale bars: (a) $20 \mu \mathrm{m}$, (b) $50 \mu \mathrm{m}$ a
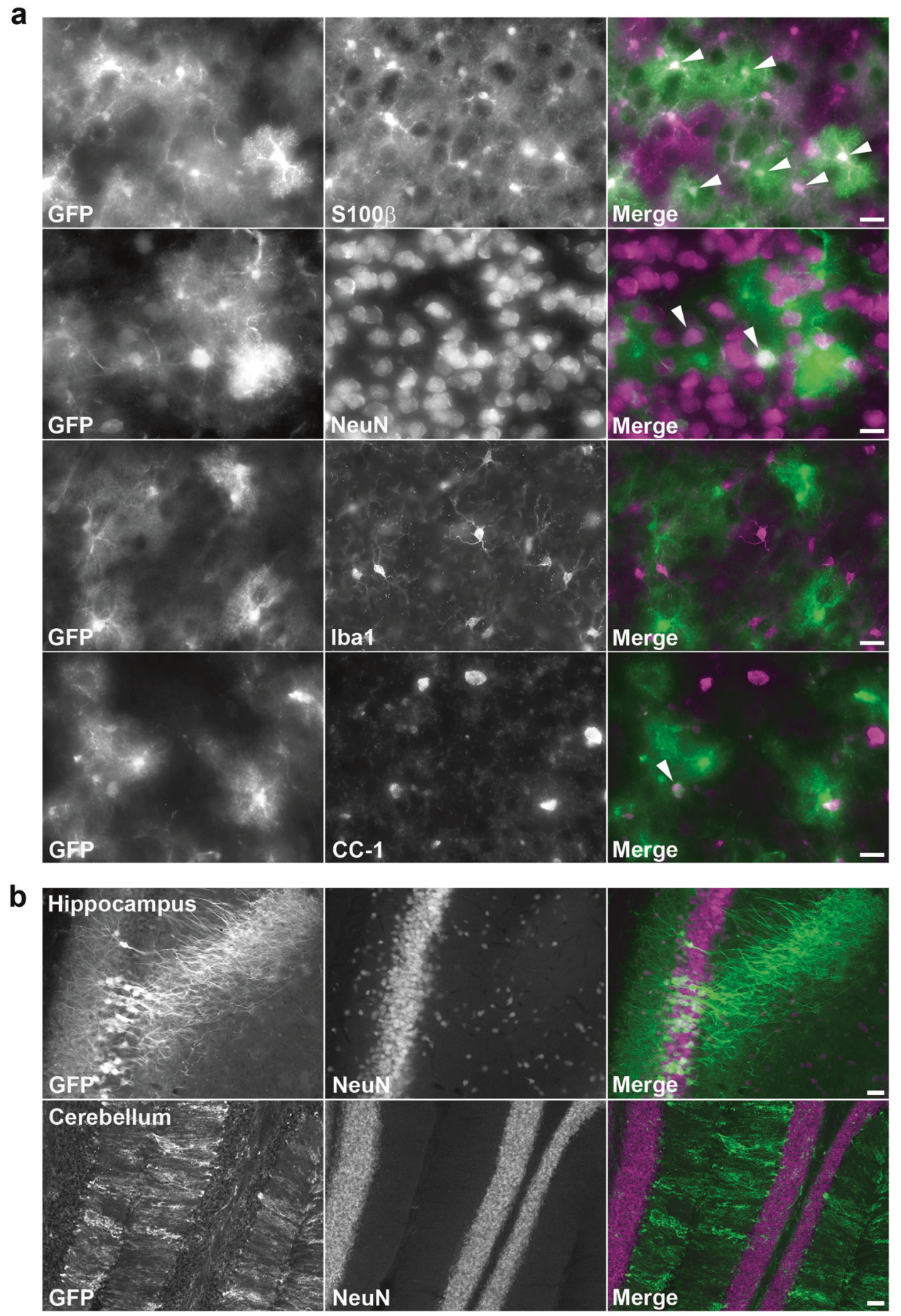

transduced astrocytes, rather than increased transduction of additional cell types.

\section{Broad neuronal expression is achieved using the human synapsin promoter}

We next assessed GFP expression in the CNS after systemic delivery of a scAAV2/PHP.B vector in which expression of
GFP was under control of the human synapsin 1 (hSYN1) promoter [20]. scAAV2/PHP.B-hSYN1-GFP was injected at two different doses $\left(2 \times 10^{11}\right.$ and $\left.1 \times 10^{12} \mathrm{vg} / \mathrm{mouse}\right)$. In direct contrast to our results using a CBA promoter, use of the hSYN1 promoter resulted almost exclusively in GFP expression in neurons, in all CNS regions evaluated (Fig. 5), including the cortex, striatum, and hippocampus (Fig. 5a-c). Importantly, GFP-positive motor neurons were 
Fig. 3 scAAV2/PHP.B in combination with a CBA promoter does not transduce motor neurons after systemic delivery. a Representative images from the lumbar spinal cord region of adult mice injected with scAAV2/9 or scAAV2/PHP.B and immunostained for GFP 3 weeks postinjection. Higher GFP expression is observed in animals injected with the scAAV2/PHP.B vector. b In the ventral horn, both vectors gave strong GFP expression in numerous cells showing typical astrocyte morphology (also see Fig. 2). However, no GFP expression in motor neurons (as judged by ChAT co-staining; magenta) was observed. c Quantification of the relative fluorescence intensities observed in spinal cord. a.u. arbitrary units. $* * p<0.01$. Scale bars: (a) $200 \mu \mathrm{m}$, (b) $50 \mu \mathrm{m}$
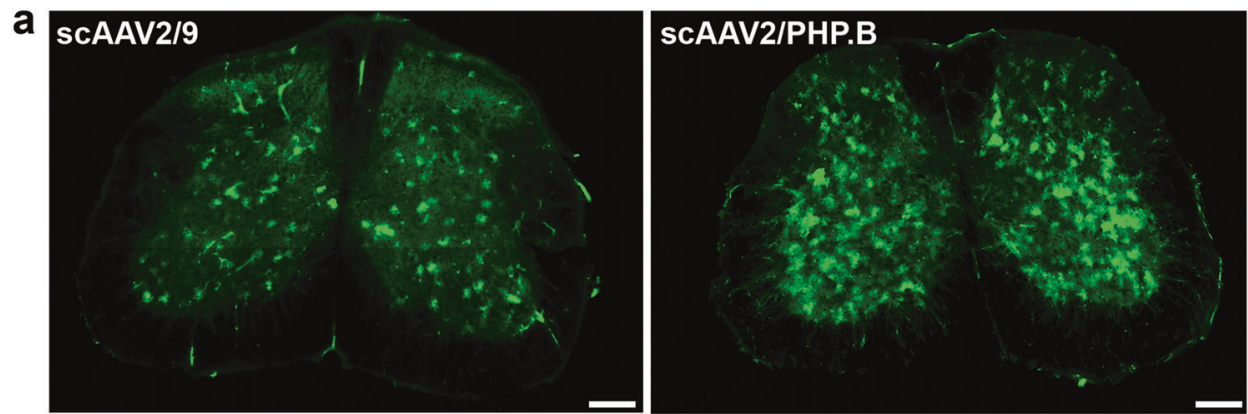

b
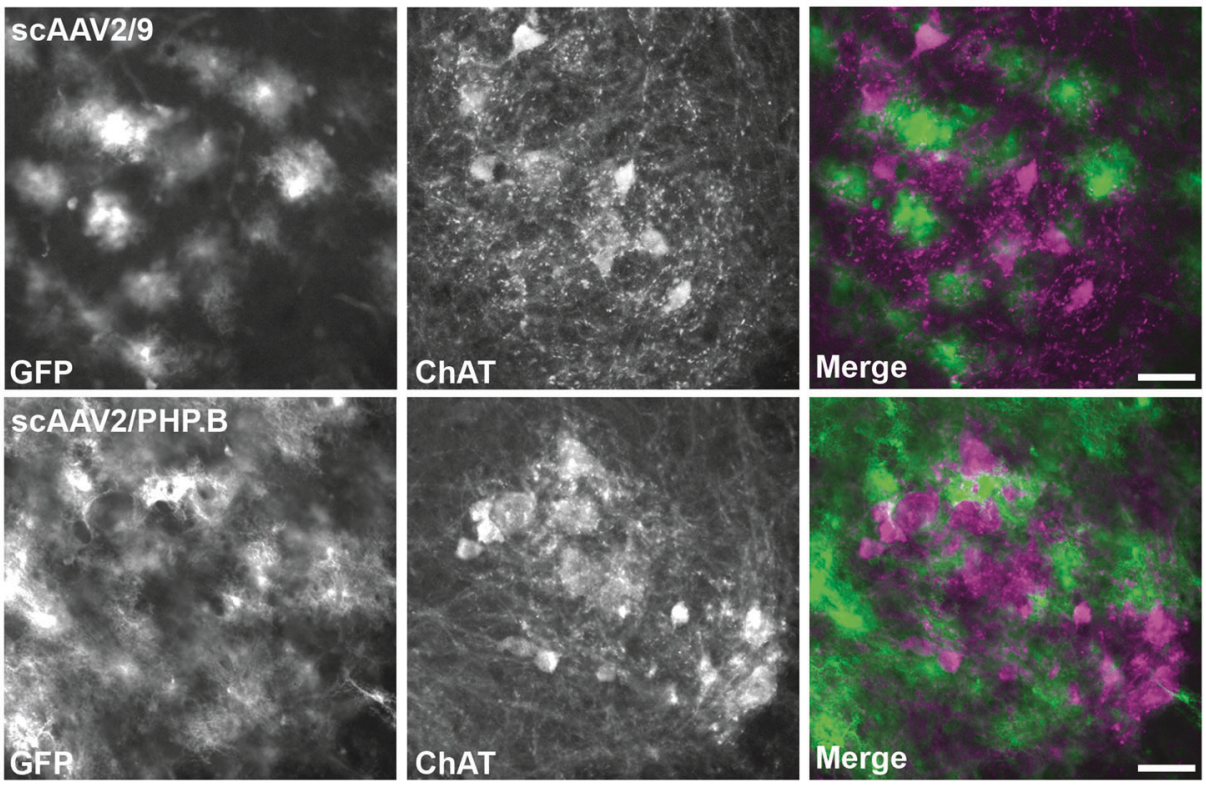

C

Spinal cord

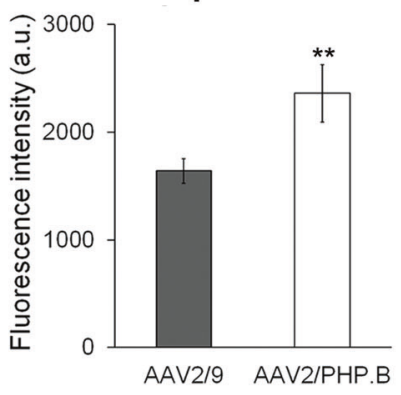

observed in spinal cord after co-labeling for ChAT and GFP (Fig. 5d). These results indicate that AAV2/PHP.B is capable of infecting the majority of cell types in the CNS, consistent with previous results [14, 18], with genome configuration and promoter choice largely determining the pattern of transgene expression.

In summary, we set out to evaluate the efficiency of CNS transduction when using systemic injection of the newly described PHP.B capsid. As expected, the PHP.B capsid with a novel combination of a self-complementary genome configuration and CBA promoter showed enhanced levels of transduction compared to ssAAV2/PHP.B, as well as
scAAV2/9. Furthermore, our results highlight the importance of choosing the correct genome configuration and promoter combination when attempting to target specific CNS cell types. First, the increase in overall transduction levels, when using a CBA promoter, was the result of an increased number of astrocytes expressing GFP, perhaps indicating the limited ability of this cell type to synthesize double-stranded DNA from a single-stranded genome. Second, the number of neurons expressing transgene remained relatively low across different regions of the cortex, irrespective of the genome configuration used, despite use of a strong ubiquitous promoter. Furthermore, 


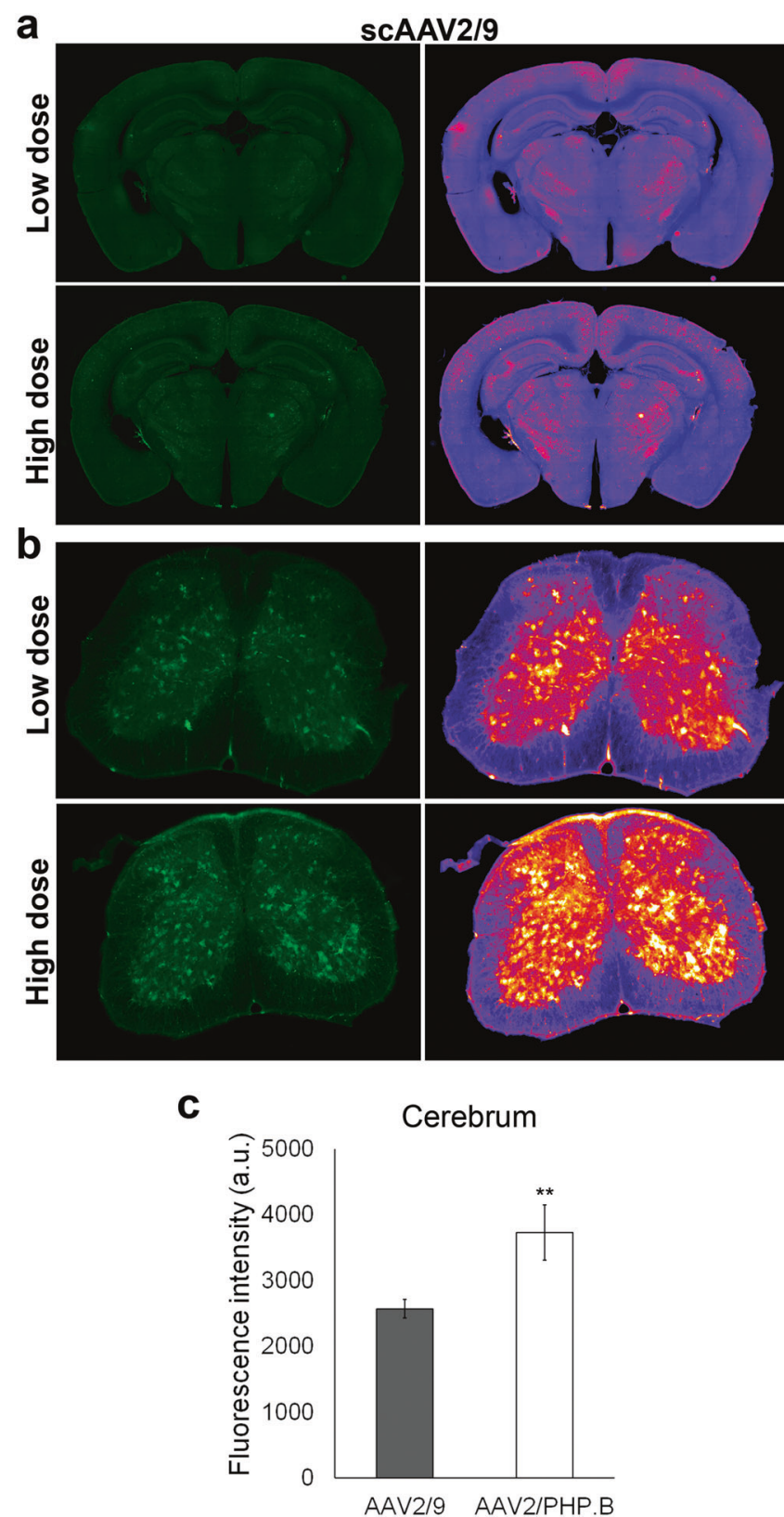

Fig. 4 Dose dependency of transgene expression with systemic delivery of scAAV2/9 or scAAV2/PHP.B vectors. Adult C57BL/6 mice received a low dose $\left(1 \times 10^{12} \mathrm{vg}\right)$ or high dose $\left(2.5 \times 10^{12} \mathrm{vg}\right)$ of scAAV2/9 or scAAV2/PHP.B vectors. Animals were euthanized 3 weeks later and GFP expression was analyzed by immunofluorescence in (a) the brain and (b) the spinal cord. For easy visual

no staining of motor neurons in the spinal cord was detected (in contrast to previous reports using a single-stranded genome and a variant of the CBA promoter) [18]. In contrast, we did observe strong neuronal expression when the hSYN1 promoter was used. These factors obviously need to be accounted for in future experiments using AAV2/PHP.B for CNS applications.

Taken together, however, our data unequivocally point towards capsid optimization as a viable strategy for
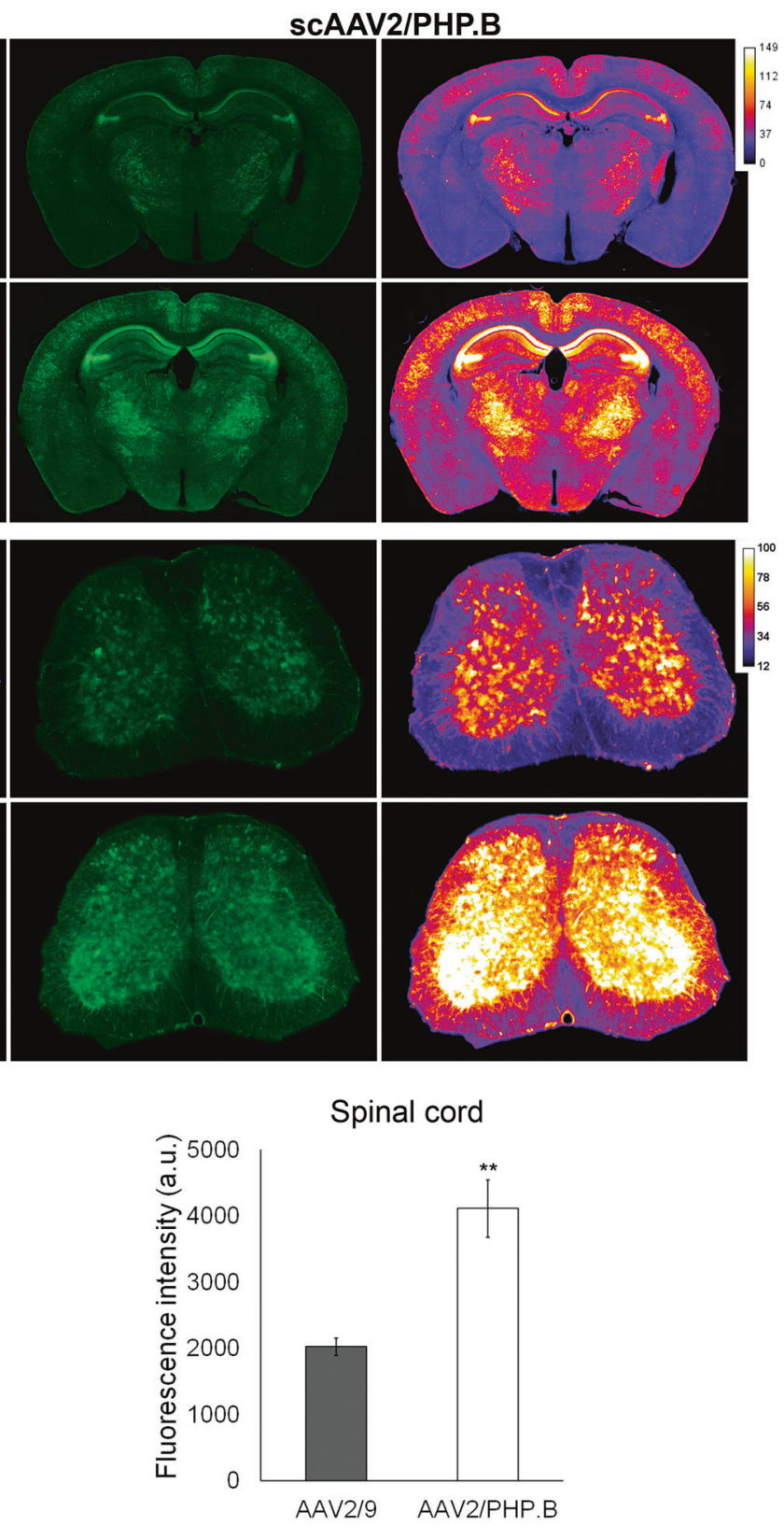

comparison of GFP intensities across the various conditions, heat maps were generated. c Quantification of the relative fluorescence intensities observed in the brain and spinal cord (Brain: 10 sections per mouse; Spinal cord: 15 sections per mouse; 3 mice per group analyzed). a.u. arbitrary units. $* * p<0.01$

improving transgene expression in CNS, following systemic delivery of scAAV-based vectors. We anticipate this will be critical for future applications of gene therapy to treat CNS disorders. Crucially, more efficient BBB crossing will allow the use of lower vectors doses, with corresponding benefits for reduction of expression in off-target tissues, reduced immunological complications arising from the use of high AAV doses, and reduced manufacturing costs. Additional modifications, associated with enhanced transcriptional 
Fig. 5 Widespread transgene expression in neurons is achieved when using the hSYN1 promoter. C57BL/6 mice received tail vein injections of a scAAV2/PHP.B vector $\left(1 \times 10^{12}\right.$ $v g$ ) expressing GFP under control of the hSYN1 promoter. GFP expression was analyzed 3 weeks postinjection. a Representative brain sections showing widespread expression of GFP. b In the cortex, strong GFP expression (green) was observed exclusively in neuronal cells (NeuN, magenta). c Representative images from the hippocampus showing strong neuronal GFP expression in the $\mathrm{CA} 3$ region. $\mathbf{d}$ In the lumbar ventral horn, GFP and ChAT costaining indicates substantial transduction of motor neurons. Scale bars: (a) $1000 \mu \mathrm{m},(\mathbf{b}-\mathbf{d})$ $50 \mu \mathrm{m}$
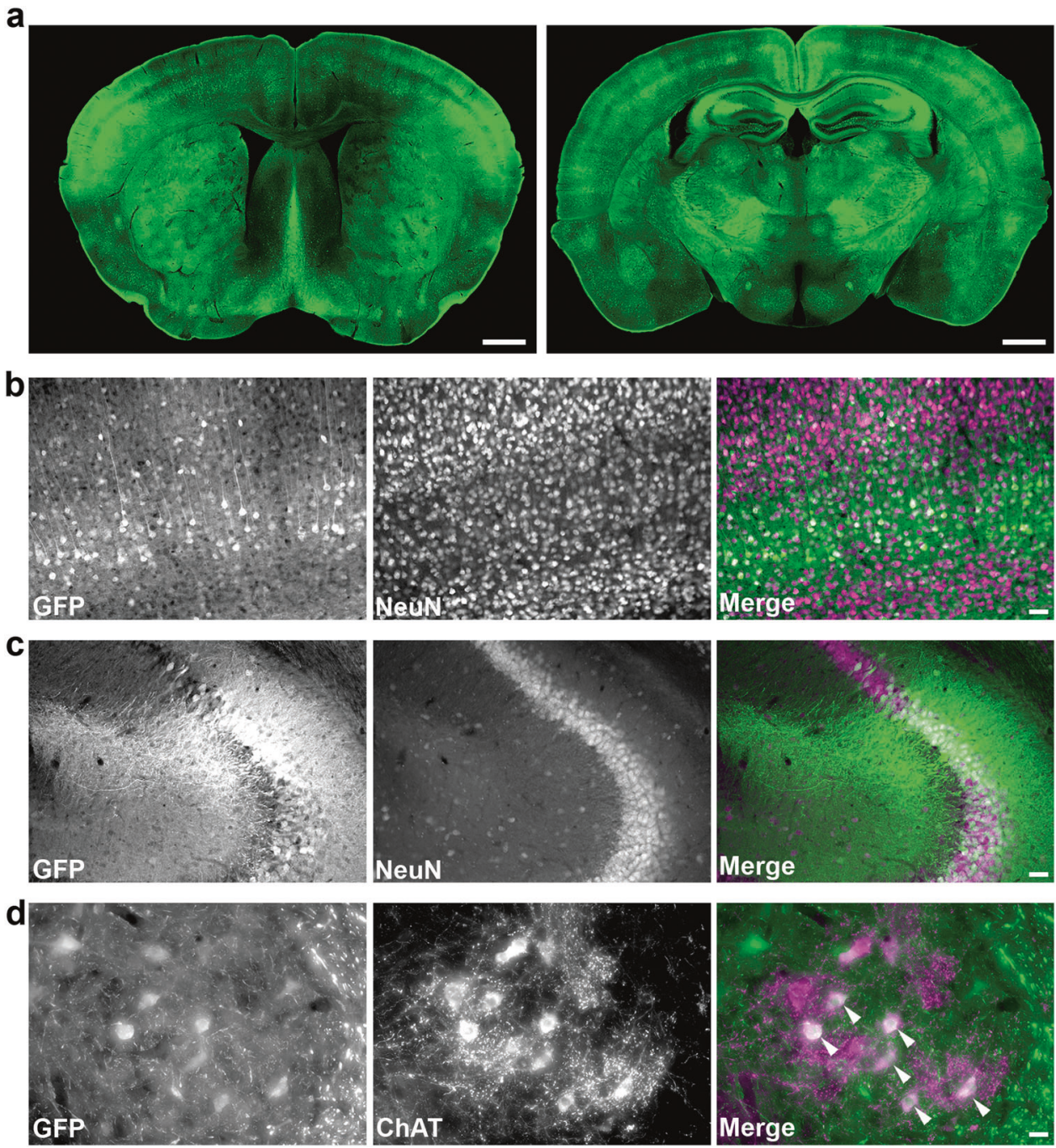

activity, are likely to further boost vector performance, allowing therapeutic levels of transgene expression to be more easily achieved. Such modifications, which include the use of highly specific CNS promoters [21], or the use of specific enhancer sequences [22-24], are necessary to further improve vector specificity and strength.

\section{Materials and methods}

\section{AAV vector production and purification}

ssAAV2/PHP.B and scAAV2/PHP.B vectors used in experiments for direct comparison of these two vector configurations (Supplementary figures 1 and 2) were produced and vector titer measured by ViGene Biosciences (Rockville, MD, USA). The AAVRep2CapPHP.B plasmid sequence was cloned in house using online data as a guide [18], and provided under a Material Transfer Agreement (MTA). Further details about packaging and purification strategies can be found on the company website (http://www.vigenebio.com). scAAV2/PHP.B and scAAV2/9 vectors used in all other experiments were prepared in the VIB core facility, using methods described previously [25]. Briefly, HEK293T cells were tritransfected with an adenovirus helper plasmid (pAd $\Delta \mathrm{F} 6)$, a packaging plasmid (AAVRep2Cap9 or AAVRep2CapPHP.B), and a scAAV transgene plasmid. Vectors were purified using an iodixanol density-gradient strategy. Vector titers were determined by quantitative PCR (expressed as $\mathrm{vg} / \mathrm{ml}$ ) and purity assessed by SDS-PAGE and silver staining.

\section{Animal procedures}

All animal procedures were approved by the animal ethics committee of the KU Leuven. Six weeks old C57BL/6 mice were injected $(100 \mu$ l total volume) in the lateral tail vein with AAV vectors at doses of either $2 \times 10^{11}, 1 \times 10^{12}$ or $2.5 \times 10^{12} \mathrm{vg} /$ mouse ( $n=3$ mice per group). In the absence 
of data quantifying the effect size, the sample size was chosen based on that used in comparable studies $[14,18]$. The entire project was conducted using female C57BL/6 J mice (Jackson Laboratory, Bar Harbor, ME). Mice were selected randomly for injection with a given serotype. The researcher that performed tail vein injection was masked to the AAV serotype injected. Further analysis was done in an unmasked manner. Three weeks postinjection, mice were transcardially perfused with phosphate buffered saline (PBS), followed by ice-cold 4\% paraformaldehyde (PFA) in PBS. Brains and spinal cords were recovered and underwent further post-fixation by overnight incubation in the same fixative, before cutting into $50 \mu \mathrm{m}$ sections on a vibratome (Leica VT1000S). In an additional set of experiments, 6 weeks old C57BL/6 mice were stereotaxically injected directly into the left cerebral ventricle (coordinates from bregma $-0.2 \mathrm{~cm}$ lateral, $+1 \mathrm{~cm}$ anteroposterior, $-2.5 \mathrm{~cm}$ depth), using a Hamilton syringe $7001 \mathrm{~N}$ ( $n=3$ per group). scAAV2/9-CBA-GFP or scAAV2/PHP.B-CBA-GFP were injected at a dose of $4 \times 10^{9} \mathrm{vg} / \mathrm{mouse}$ (total injected volume $1.8 \mu \mathrm{l})$. Three weeks postinjection, mice were euthanized and brain samples were collected for histology. Samples from all injected animals were included for analysis.

\section{Immunohistochemistry}

Sections were rinsed twice in PBS, before blocking for $1 \mathrm{~h}$ at room temperature in $1 \%$ Triton X-100 in PBS, supplemented with $10 \%$ normal donkey serum (NDS). Primary antibody labeling was done overnight at $4{ }^{\circ} \mathrm{C}$. Antibodies used were: rabbit anti-GFP (1:300, Synaptic Systems, \#132002), guinea pig anti-NeuN (1:400, Synaptic Systems, \#266004), mouse anti-S100ß (1:1000, Sigma, \#S2532), goat anti-ChAT (1:200, Merck Millipore, \#AB144P), mouse anti-CC-1 (1:200, Abcam, \#ab16794) and rabbit anti-Iba1 (1:600, Synaptic Systems, \#234003). Sections were then washed in PBS before incubation with secondary antibodies (diluted in $1 \%$ Triton X-100/PBS) for $2-3 \mathrm{~h}$ at room temperature. Secondary antibodies used were: donkey anti-rabbit Alexa Fluor 488 (1:1000, Invitrogen, \#A21206), donkey anti-guinea pig Cy3 (1:200, Jackson Immuno, \#706-165-148), and donkey anti-mouse Cy5 (1:200, Jackson Immuno, \#715-175-150). Sections were washed before mounting with Fluoromount-G containing DAPI.

For manual counting of double-positive cells, images were taken on a Leica DM5500 epifluorescence microscope, equipped with an APO10x/NA 0.40 objective. Cells were counted in 60 different observation fields, taken from across the cerebral cortex (10x views per mouse, $n=3$ per group). Measurements of total GFP fluorescence were made from wide field images taken on a Zeiss Slide scanner (Axio Scan Z1), equipped with a PL APO20x/NA 0.8 objective, controlled using Zen 2 software. Fluorescence intensity measurements were made using ZEN lite analysis software (Zeiss). Intensities are reported in arbitrary units (following background correction).

\section{Statistical analysis}

Data are expressed as mean \pm s.d. Differences between vector groups were analyzed using a one-way ANOVA test, followed by a post hoc two-tailed Student's $t$-test. $p$ values $<0.05$ were considered significant. Sample groups were all compared to a relevant control group. Data in each experimental group was normally distributed and variance between the groups was similar.

Acknowledgements M.R. is a postdoctoral researcher with the FWO $(133722 / 1204517 \mathrm{~N})$, and acknowledges the continuous support of the Fundación Cardiovascular de Colombia. S.D. was supported by The Foundation for Alzheimer Research (SAO-FRA) (P\#14006). This work was supported by grants from the Thierry Latran Foundation (SOD-VIP), FWO (1513616N), European Research Council (ERC) Starting Grant (AstroFunc: 281961) and Proof of Concept Grant (ADVIP: 713755) to MGH. FDV and MGH acknowledge Michael Dunlop and Ian Hutchinson.

\section{Compliance with ethical standards}

Conflict of interest The authors declare that they have no conflict of interest.

\section{References}

1. Hocquemiller M, Giersch L, Audrain M, Parker S, Cartier N. Adeno-associated virus-based gene therapy for CNS diseases. Hum Gene Ther. 2016;27:478-96.

2. Gray SJ, Nagabhushan Kalburgi S, McCown TJ, Jude Samulski R. Global CNS gene delivery and evasion of anti-AAVneutralizing antibodies by intrathecal AAV administration in non-human primates. Gene Ther. 2013;20:450-9.

3. Cohen-Pfeffer JL, Gururangan S, Lester T, Lim DA, Shaywitz AJ, Westphal M, et al. Intracerebroventricular delivery as a safe, longterm route of drug administration. Pediatr Neurol. 2017;67:23-35.

4. Duque S, oussemet B, Riviere C, Marais T, Dubreil L, Douar A$\mathrm{M}$, et al. Intravenous administration of self-complementary AAV9 enables transgene delivery to adult motor neurons. Mol Ther J Am Soc Gene Ther. 2009;17:1187-96.

5. Foust KD, Nurre E, Montgomery CL, Hernandez A, Chan CM, Kaspar BK. Intravascular AAV9 preferentially targets neonatal neurons and adult astrocytes. Nat Biotechnol. 2009;27:59-65.

6. Iwata N, Sekiguchi M, Hattori Y, Takahashi A, Asai M, Ji B, et al. Global brain delivery of neprilysin gene by intravascular administration of AAV vector in mice. Sci Rep. 2013;3:1472.

7. Garg SK, Lioy DT, Cheval H, McGann JC, Bissonnette JM, Murtha MJ, et al. Systemic delivery of MeCP2 rescues behavioral and cellular deficits in female mouse models of Rett syndrome. J Neurosci J Soc Neurosci. 2013;33:13612-20.

8. Foust KD, Salazar DL, Likhite S, Ferraiuolo L, Ditsworth D, Ilieva $\mathrm{H}$, et al. Therapeutic AAV9-mediated suppression of mutant SOD1 slows disease progression and extends survival in models of inherited ALS. Mol Ther J Am Soc Gene Ther. 2013;21:2148-59.

9. Foust KD, Wang X, McGovern VL, Braun L, Bevan AK, Haidet $\mathrm{AM}$, et al. Rescue of the spinal muscular atrophy phenotype in a 
mouse model by early postnatal delivery of SMN. Nat Biotechnol. 2010;28:271-4.

10. McCarty DM, Fu H, Monahan PE, Toulson CE, Naik P, Samulski RJ. Adeno-associated virus terminal repeat (TR) mutant generates self-complementary vectors to overcome the rate-limiting step to transduction in vivo. Gene Ther. 2003;10:2112-8.

11. Dashkoff J, Lerner EP, Truong N, Klickstein JA, Fan Z, Mu D, et al. Tailored transgene expression to specific cell types in the central nervous system after peripheral injection with AAV9. Mol Ther Methods Clin Dev. 2016;3:16081.

12. Gray SJ, Matagne V, Bachaboina L, Yadav S, Ojeda SR, Samulski RJ. Preclinical differences of intravascular aav9 delivery to neurons and glia: a comparative study of adult mice and nonhuman primates. Mol Ther. 2011;19:1058-69.

13. Gray SJ, Foti SB, Schwartz JW, Bachaboina L, Taylor-Blake B, Coleman J, et al. Optimizing promoters for recombinant adenoassociated virus-mediated gene expression in the peripheral and central nervous system using self-complementary vectors. Hum Gene Ther. 2011;22:1143-53.

14. Jackson KL, Dayton RD, Deverman BE, Klein RL. Better Targeting, Better Efficiency for Wide-Scale Neuronal Transduction with the Synapsin Promoter and AAV-PHP.B. Front Mol Neurosci. 2016;9:116.

15. A Kotterman M, Schaffer DV. Engineered AAV vectors for improved central nervous system gene delivery. Neurogenesis. 2015;2:e1122700 https://doi.org/10.1080/23262133.2015.1122700

16. Pulicherla N, Shen S, Yadav S, Debbink K, Govindasamy L, Agbandje-McKenna M, et al. Engineering liver-detargeted AAV9 vectors for cardiac and musculoskeletal gene transfer. Mol Ther J Am Soc Gene Ther. 2011;19:1070-8.

17. Choudhury SR, Harris AF, Cabral DJ, Keeler AM, Sapp E, Ferreira JS, et al. Widespread central nervous system gene transfer and silencing after systemic delivery of novel AAV-AS vector. Mol Ther J Am Soc Gene Ther. 2016;24:726-35.
18. Deverman BE, Pravdo PL, Simpson BP, Kumar SR, Chan KY, Banerjee A, et al. Cre-dependent selection yields AAV variants for widespread gene transfer to the adult brain. Nat Biotechnol. 2016;34:204-9.

19. Hudry E, Martin C, Gandhi S, György B, Scheffer DI, Mu D, et al. Exosome-associated AAV vector as a robust and convenient neuroscience tool. Gene Ther. 2016;23:380-92.

20. Kügler S, Kilic E, Bähr M. Human synapsin 1 gene promoter confers highly neuron-specific long-term transgene expression from an adenoviral vector in the adult rat brain depending on the transduced area. Gene Ther. 2003;10:337-47.

21. Gray SJ, Foti SB, Schwartz JW, Bachaboina L, Taylor-Blake B, Coleman J, et al. Optimizing promoters for recombinant adenoassociated virus-mediated gene expression in the peripheral and central nervous system using self-complementary vectors. Hum Gene Ther. 2011;22:1143-53.

22. Rincon MY, Sarcar S, Danso-Abeam D, Keyaerts M, Matrai J, Samara-Kuko E, et al. Genome-wide computational analysis reveals cardiomyocyte-specific transcriptional Cis-regulatory motifs that enable efficient cardiac gene therapy. Mol Ther $\mathrm{J}$ Am Soc Gene Ther. 2015;23:43-52.

23. Chuah MK, Petrus I, De Bleser P, Le Guiner C, Gernoux G, Adjali O, et al. Liver-specific transcriptional modules identified by genome-wide in silico analysis enable efficient gene therapy in mice and non-human primates. Mol Ther J Am Soc Gene Ther. 2014;22:1605-13.

24. Nair N, Rincon MY, Evens H, Sarcar S, Dastidar S, Samara-Kuko E, et al. Computationally designed liver-specific transcriptional modules and hyperactive factor IX improve hepatic gene therapy. Blood. 2014;123:3195-9.

25. Duque S, Joussemet B, Riviere C, Marais T, Dubreil L, Douar A$\mathrm{M}$, et al. Intravenous administration of self-complementary AAV9 enables transgene delivery to adult motor neurons. Mol Ther J Am Soc Gene Ther. 2009;17:1187-96. 